\title{
Whistler-Mode Electron Cyclotron Emission from the Phaedrus-B End Cell
}

\author{
BINSHENG CUI, PAUL H. PROBERT, PETER D. BROOKER, PAUL D. NONN, DOUGLAS L. KEIL, \\ NOAH HERSHKOWITZ, SENIOR MEMBER, IEEE, RICHARD P. MAJESKI, ROBERT A. BREUN, AND \\ RICHARD F. ELLIS
}

\begin{abstract}
Measurements of whistler-mode electron cyclotron emission have been made in the Phaedrus-B tandem mirror end cell. The receiving horn was located on the axis of the machine just outboard of the end cell. Under normal conditions, the signal had a component which was identified as blackbody emission, and an electron temperature was obtained which was in good agreement with electron end loss energy-analyzer data. In addition, the signal contained short bursts of unknown origin. Significant heating of the electrons due to end-cell ICRF was seen. Comparison of end loss and Thomson scattering data showed that emission from hotter edge electrons was responsible for a large part of the total signal.
\end{abstract}

\section{INTRODUCTION}

$\mathrm{E}$ LECTRON CYCLOTRON emission has been used as a nonperturbing diagnostic for measuring electron temperature in many experiments [1]-[4]. In magnetic mirror devices, whistler-mode electron cyclotron emission (WECE), propagating parallel to the magnetic field, has been studied [5]-[8]. In many of these experiments the emission has been from hot electrons or has been associated with instabilities [8]-[10]. In TMX-U, for example, bursts of emission were observed which were attributed to instabilities related to hot and/or anisotropic electron distributions produced by strong end-cell electron cyclotron heating $(\mathrm{ECH})[8]$.

The reason for using the whistler mode is that the emission can be strongly blackbody in origin, simplifying the calculation of the electron temperature and, in a magnetic mirror geometry, the whistler mode can propagate up the magnetic field gradient at arbitrarily high plasma densities [7]. The end-cell electron temperature is an important parameter in thermal-barrier tandem mirrors such as Phaedrus-B. Localized heating of electrons in the end cell can create a positive potential relative to the central cell, leading to enhanced confinement of central-cell ions [11], [12].

In this paper, we present the results of WECE mea-

Manuscript received June 23, 1988; revised September 29, 1988. This work was supported by the U.S. Department of Energy under Grant DEFG02-88ER53264

B. Cui, P. H. Probert, P. D. Brooker, P. D. Nonn, D. L. Keil, N. Hershkowitz, R. P. Majeski, and R. A. Breun are with the Department of Nuclear Engineering and Engineering Physics, University of WisconsinMadison, Madison, WI 53706.

R. F. Ellis is with the Laboratory for Plasma and Fusion Energy Studies, University of Maryland, College Park, MD 20742.

IEEE Log Number 8825120 . surements from the Phaedrus-B tandem mirror end cell during heating with electromagnetic waves in the ion cyclotron frequency range (ICRF) and with ECH. Signals associated with both thermal emission and bursts of unknown origin were observed. In Section II, we describe the experimental arrangement. In Section III, we present and analyze the emission data. In Section IV, we give the resulting electron temperature and compare this with data from other diagnostics. Finally, in Section V, we present our conclusions.

\section{Experimental Arrangement}

Phaedrus-B is a five-cell tandem mirror consisting of a $0.08 \mathrm{~T}$ central cell bounded by $0.75 \mathrm{~T}$ choke coils, followed by $0.16 \mathrm{~T}$ (minimum) thermal barrier cells which are in turn bounded by quadrupole end cells which have a midplane field of $0.3 \mathrm{~T}$ and a mirror ratio of 1.7 . The central cell plasma radius is $16 \mathrm{~cm}$. Plasma is produced, heated, and stabilized using ICRF power of approximately $400 \mathrm{~kW}$ [13], [14]. Start-up is achieved by electron cyclotron breakdown in the central cell using a 5 $\mathrm{GHz}, 25 \mathrm{~kW}, 3 \mathrm{~ms}$ microwave pulse [15]. Plasma densities throughout the device in these experiments are as high as $4 \times 10^{12} \mathrm{~cm}^{-3}$.

In the end cells the ratio $\omega_{p e}^{2} / \omega_{c e}^{2}$ (where $\omega_{p e}$ is the electron plasma frequency and $\omega_{c e}$ is the electron cyclotron frequency) is typically in the range from 0.4 to 4.0 , making the high-density capabilities of WECE attractive. At the end-cell midplane, the path in the perpendicular direction is obstructed by ICRF antennas which provide for the fueling and heating of the end-cell plasma, making perpendicular emission measurements difficult.

To obtain a WECE signal, a rectangular horn ( $X$ band, $8.4 \mathrm{~cm} E$ plane $\times 11.2 \mathrm{~cm} H$ plane $\times 20 \mathrm{~cm}$ long $)$ antenna was located $65 \mathrm{~cm}$ outboard from the midplane of the Phaedrus-B end cell, on the axis of the machine. The signal from this horn was brought out of the machine through standard $X$-band waveguide (cutoff frequency, $6.56 \mathrm{GHz}$ ), and the vacuum feedthrough comprised a mica window and viton $\mathrm{O}$-ring seal in a waveguide flange. The receiver system was connected to the waveguide with a 5 $m$ length of low-loss coaxial cable and was enclosed in a metal shielding box to prevent spurious radiation from the ICRF sources from leaking into the IF amplifier of the 



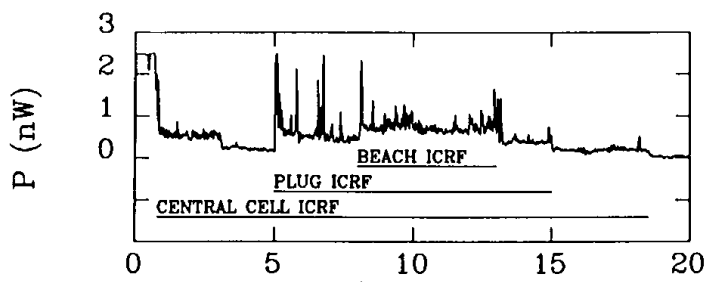

(a)

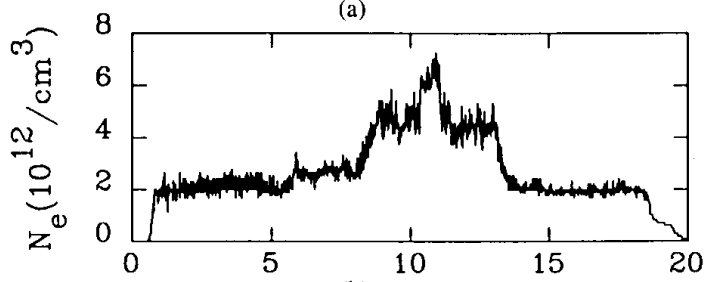

(b)

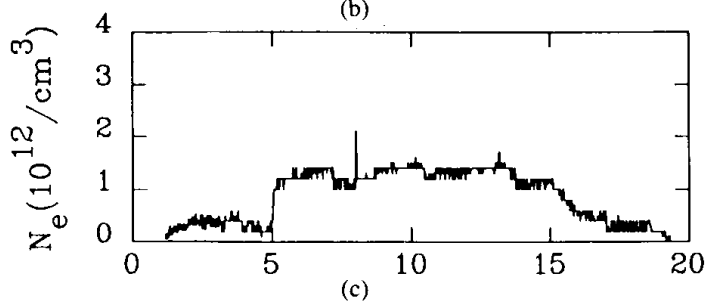

TIME(msec)

Fig. 2. (a) The WECE signal in the presence of the central-cell ICRF, the plug ICRF, and the beach ICRF. Also shown are corresponding line averaged densities from (b) the central-cell, and (c) end-cell interferometer systems.

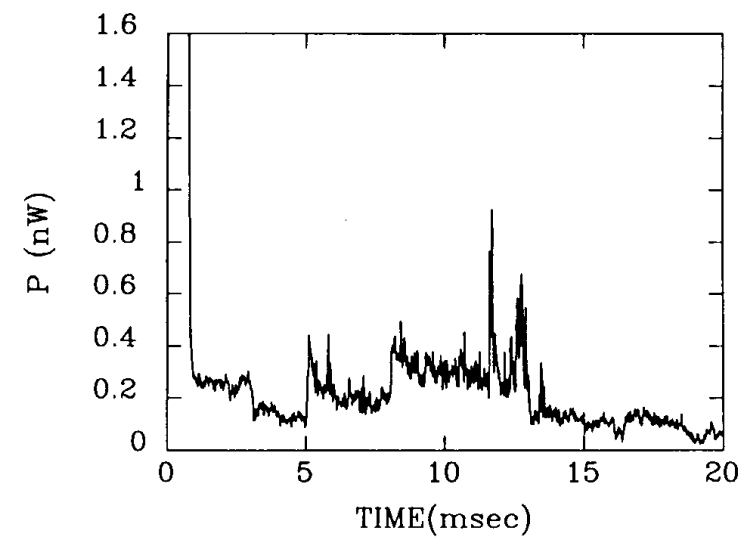

Fig. 3. The WECE signal when the local oscillator's frequency is $\sim 9.5$ $\mathrm{GHz}$ (axial position $\sim 20 \mathrm{~cm}$ off midplane), showing a reduction in the burst amplitude and frequency.

due to both end-cell RF sources even though the plug ICRF heating causes large increases in the end-cell density and the beach ICRF does not.

When the WECE signal was obtained at $\sim 9.5 \mathrm{GHz}$, corresponding to $20 \mathrm{~cm}$ from the midplane, the bursts were smaller, as shown in Fig. 3. When the receiving horn was withdrawn from the plasma, the bursts were still present and often much more intense and frequent, whereas the floor signal was much smaller. The burst signals were observed in the absence of end-cell $\mathrm{ECH}$ and so their origin cannot be easily attributed to an instability
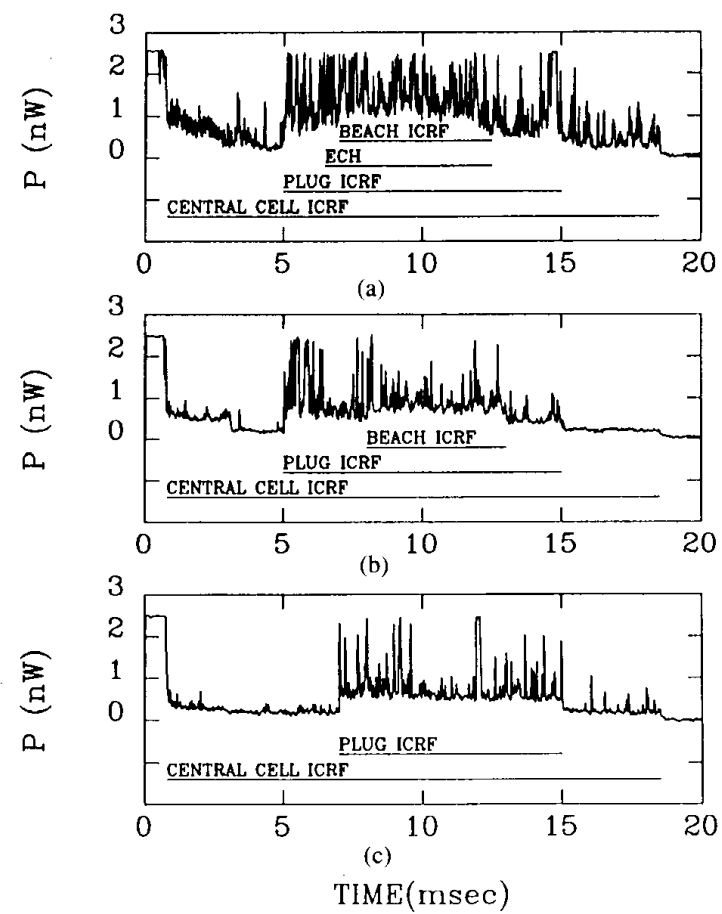

Fig. 4. WECE emission versus time for three representative discharges. (a) All (beach, plug, and central-cell) ICRF sources are present as in dicated, as well as the $14 \mathrm{GHz}$ end-cell ECH. (b) Same as in (a) except with no ECH. (c) Only plug and central-cell ICRF are present.

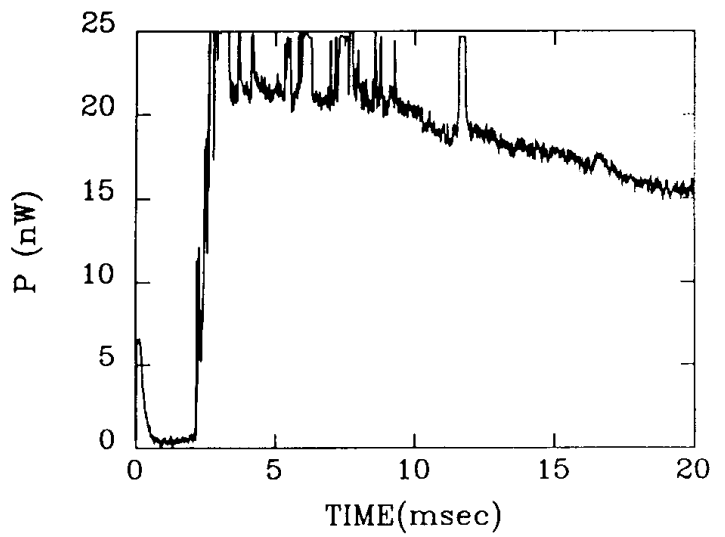

Fig. 5. The WECE signal versus time from hot electrons in the end cell during $\mathrm{ECH}$ heating where the plasma density was $<10^{11} \mathrm{~cm}^{-3}$.

driven by a strongly non-Maxwellian electron distribution.

When ECH $(10 \mathrm{~kW}$ at $14 \mathrm{GHz}$, resonant near the throats of the end cells) was present, an increase in the emission could be seen. These signals are shown in Fig. 4 and compared to similar discharges without $\mathrm{ECH}$. We also investigated hot electron cyclotron emission from the $\mathrm{ECH}$ heating in a low-density plasma. In Fig. 5, the ECH power was on from 2 to $10 \mathrm{~ms}$, though no other heating or plasma sources were present. The plasma density was below the detectable level $\left(<10^{11} \mathrm{~cm}^{-3}\right)$ and the high emission sig- 
nal (10 times greater than normal) decayed exponentially with a time constant greater than 20 ms.

\section{Electron Temperature}

The floor WECE signal was used to estimate the electron temperature. The received WECE power can be expressed in terms of the electron temperature $(\mathrm{eV})$ by [2], [5], [6]

$$
P=g T_{e}\left(1-e^{-\tau}\right) \Delta f
$$

where $\Delta f=600 \mathrm{MHz}$ is the bandwidth of the receiver. The calibration factor $g$ can be expressed as $g=g_{1} g_{2} g_{3} g_{4}$, where $g_{1}$ takes into account the effects of antenna polarization, $g_{2}$ is the attenuation in the waveguide between the antenna and the noise source, $g_{3}$ is the antenna pattern filling factor, and $g_{4}$ is the receiver's relative gain versus frequency. The optical depth $\tau$ can be approximated for the high-density $(q>\beta)$ case by [16], [7]

$$
\tau \cong \sqrt{3 \pi}(q / \beta)^{2 / 3} \beta(\omega / c) L
$$

where $q=\omega_{p e}^{2} / \omega_{c e}^{2}, \beta=\sqrt{2 T_{e} / m c^{2}}$, and $L$ is the magnetic field scale length, $L \equiv B /(d B / d z)$.

During the ICRF sustained operation the electron density was $1 \rightarrow 2 \times 10^{12} \mathrm{~cm}^{-3}$ and the electron temperature was typically $20 \sim 40 \mathrm{eV}$. The optical depth as a function of axial position for these parameters is graphed in Fig. 6. It is much greater than 1.0 everywhere, indicating that the emission should be blackbody.

In principle, WECE can provide an accurate measurement of the source position because the frequency is associated with a unique magnetic field and hence axial location. However, the presence of Doppler broadening, finite optical depth, and finite receiver bandwidth all limit the spatial resolution. Doppler broadening, when the optical depth $\tau$ is large, can result in a shift of the source point to a higher $B$ field. This shift can be expressed as [7]

$$
\begin{aligned}
\Delta z & \cong \Delta f_{d} L / f \\
\Delta f_{d} & \cong f \beta N_{r}=f \beta(\sqrt{3} / 2)(\sqrt{\pi} q q / \beta)^{1 / 3} \\
& \cong 600 \mathrm{MHz}
\end{aligned}
$$

where $N_{r}$ is the plasma index of refraction and $f$ is the receiver frequency. For Phaedrus- $\mathrm{B}$, this gives $\Delta z \sim 8$ $\mathrm{cm}$, which we note is the same as the spatial resolution due to the finite receiver bandwidth.

The receiver power was converted to an electron temperature by calibration against a standard noise source. The electron temperature is simply expressed by

$$
T_{e}(z)=\xi T_{n} /\left(g_{1} g_{2} g_{3} g_{4}\right)
$$

where $T_{n}$ is the noise temperature of the calibration standard (about $0.852 \mathrm{eV}$ ) and $\xi$ is the receiver output normalized to its response to the noise source standard at a frequency of $8.64 \mathrm{GHz}$.

The whistler propagating parallel to the magnetic field is circularly polarized while the antenna is linearly polarized, so $g_{1}=0.5$. The measured attenuation from the an-

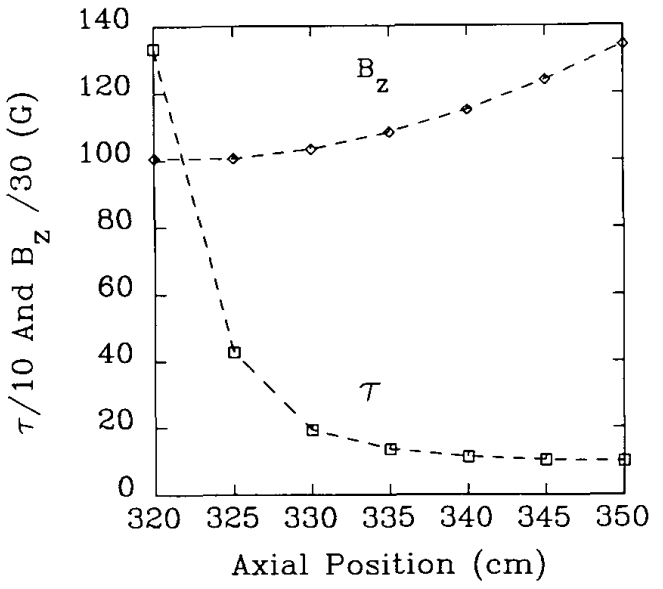

Fig. 6. The plasma optical depth for the WECE signal versus axial position from equation (2). Parameters were $n_{e}=10^{12} \mathrm{~cm}^{-3}, T_{e}=20 \mathrm{eV}$. Also shown is the magnetic field.

tenna to the switch was $g_{2}=0.8$. The geometry factor $g_{3}$, which is a measure of the fraction of the antenna's radiation pattern which is filled by the source, was estimated by measuring the emission from a fluorescent lamp (which acts as a broadband, spatially distributed noise source) masked by a series of aluminum-foil baffles which had openings cut in them which corresponded to the Phaedrus-B end-cell plasma cross section. The results of these measurements, relative to the emission of the unmasked lamp, for three different axial locations are shown in Fig. 7. These values must be considered to be only an estimate of $g_{3}$ because i) the tests were conducted in free space rather than in a magnetized plasma. Significant refraction effects can be expected because, in the Phaedrus-B end cell, the ratio of the plasma frequency to the receiver frequency is of order unity, and ii) the behavior of the waves inside the horn as they reflect from the metal surfaces and travel to the waveguide is difficult to calculate in the presence of plasma.

Finally, $g_{4}$, measured by varying the local oscillator frequency while using the calibrated noise source, is also shown in Fig. 7. The receiver response versus noise source temperature (see Fig. 8) shows that the response of the receiver is approximately linear over the frequency range used in these experiments.

In Fig. 9 we show the resulting electron temperature calculated from the WECE floor signal using (5) and averaging a series of 24 shots. Electron temperature as a function of axial position near the end-cell midplane was obtained by changing the local oscillator's frequency from discharge to discharge, and the results are shown in Fig. 10. The electron temperature is seen to be independent of axial position from 320 to $336 \mathrm{~cm}$. These data were not corrected for a Doppler broadening shift of source position because no axial $T_{e}$ variations were seen.

The other electron temperature diagnostics in the Phaedrus- $B$ end cell were Thomson scattering on the axis of the east end-cell midplane, and gridded-endless-energy 


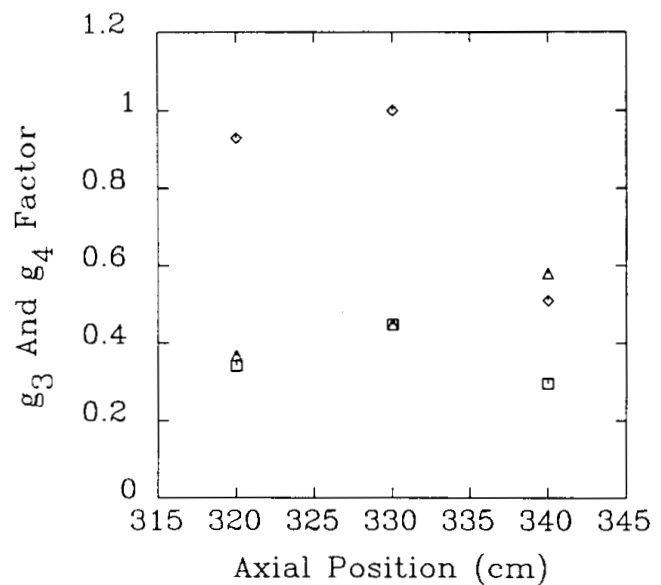

Fig. 7. The filling factor $g_{3}$ (triangles), the frequency dependency of the receiver gain $g_{4}$ (diamonds), and the product of the two (squares) versus axial position in the Phaedrus-B end cell.

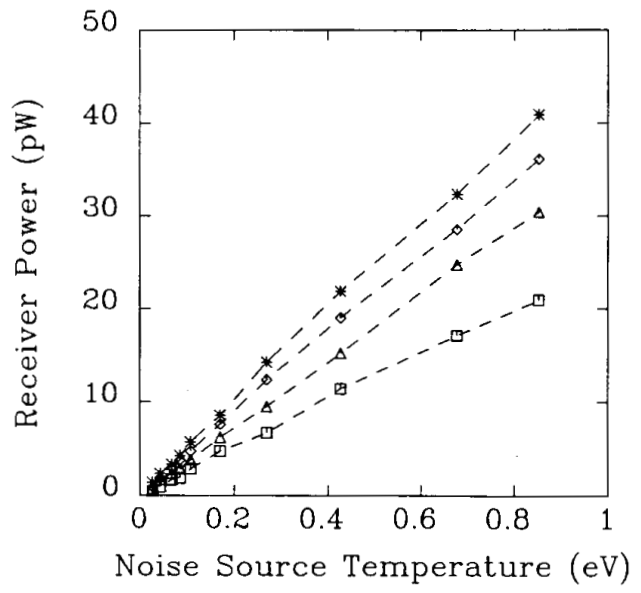

Fig. 8. The receiver output signal calibration using the noise source versus local oscillator's frequency. The frequencies were 8.64 (stars), 8.88 (diamonds), 9.22 (triangles), and 9.62 (squares) $\mathrm{GHz}$.

analyzers mounted on the end walls. A comparison of the electron temperature from WECE with the electron temperature from Thomson scattering, given in Fig. 11, shows that the temperature from WECE was generally higher and was uncorrelated with the Thomson scattering's shot-to-shot variations. We can understand this apparent discrepancy by considering the end loss data (see Fig. 12) which show that the spatial distribution of the electron temperature is very hollow and nonaxisymmetric (which we believe is due to nonuniform $E_{z}$ fields from the ICRF antennas). These measurements explain the disagreement between Thomson scattering and WECEThomson scattering only gives the on-axis value, which is low (on the order of $20 \mathrm{eV}$ and less dependent on the end-cell ICRF power), and WECE gives an average of the electron temperature over the plasma cross section, which in this case is dominated by the edge.

The electron temperatures determined by WECE and

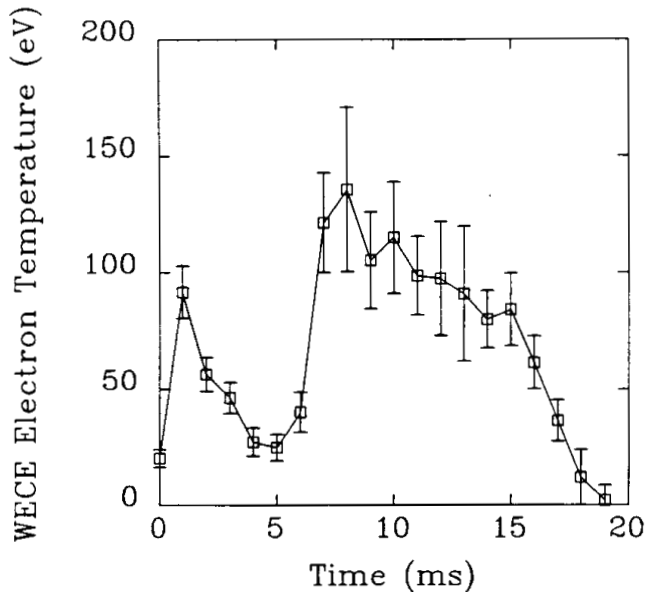

Fig. 9. The WECE-derived electron temperature calculated from the floor emission signal and equation (5), from an average over 24 shots. The error bars are the standard deviations of this average and represent shotto-shot variations in the signal. Here the central-cell ICRF was on from 0.5 to $17.5 \mathrm{~ms}$, the plug ICRF was on from 6 to $16 \mathrm{~ms}$, and the beach ICRF was on from 7 to $12 \mathrm{~ms}$. The signal from 0 to $3 \mathrm{~ms}$ contains spurious emission from the startup $\mathrm{ECH}$

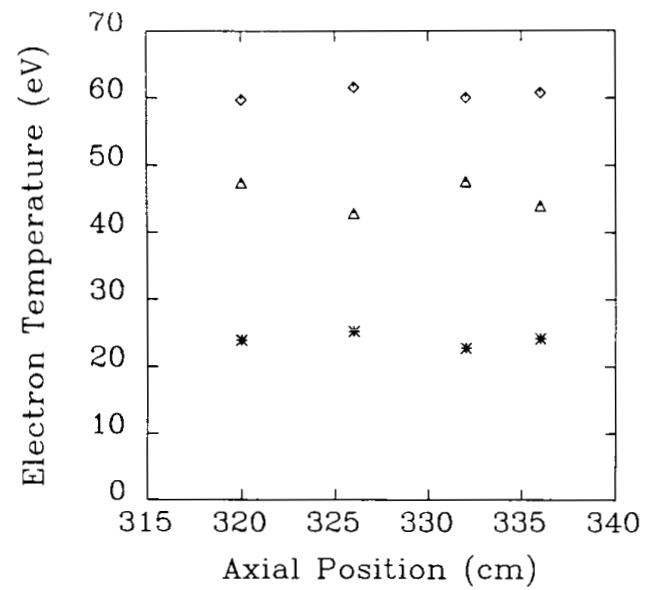

Fig. 10. The WECE-derived electron temperature versus axial position. The diamonds are for all (beach, plug, and central-cell) ICRF sources operating, the triangles for plug and central-cell ICRF, and the asterisks are for central-cell ICRF only.

by spatially averaging over the electron end loss temperature are compared in Fig. 13. It should be noted that the end loss temperature tends to weigh higher temperature components more heavily than lower temperature components and so should give a higher value of $T_{e}$. Also, the end loss data give a measure of parallel temperature, whereas WECE should be sensitive to the perpendicular temperature. In view of this, we find the agreement shown in Fig. 13 to be remarkable.

We note that the emission signals shown in Fig. 2 seem to show instantaneous changes in electron temperature as the plug and beach ICRF sources are turned on and off. Closer examination of the signals shows that the rise and decay times of the signals are from 20 to $50 \mu \mathrm{s}$, compa- 


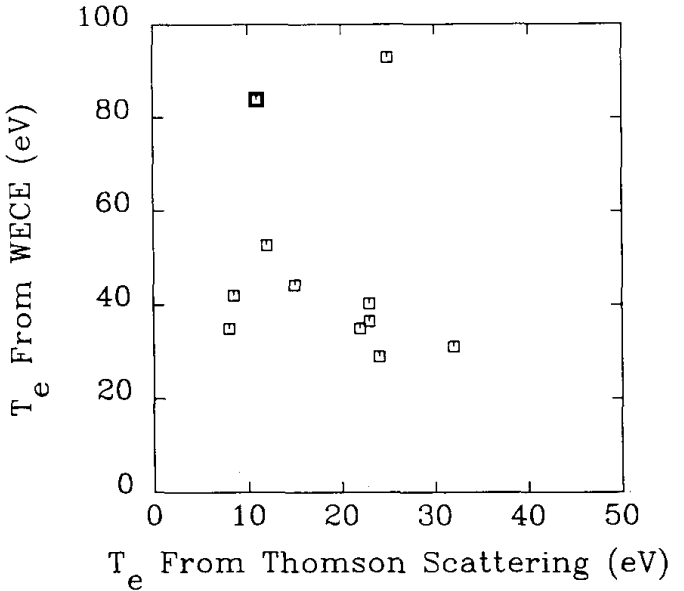

Fig. 11. The electron temperature from WECE versus the electron temperature from Thomson scattering for the case of simultaneous beach and plug ICRF heating.

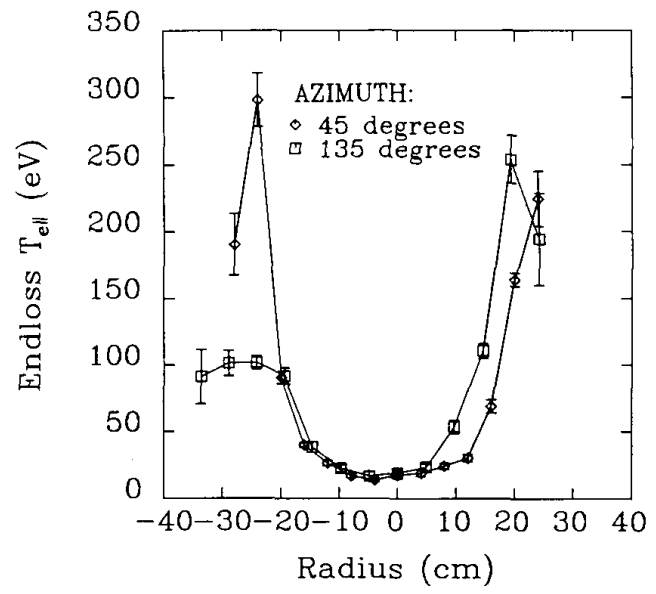

Fig. 12. The radial profile of the east end loss-energy-analyzer electron temperature for the case of simultaneous beach and plug ICRF heating for two different azimuthal angles. The radial scale is such that a radius of $25 \mathrm{~cm}$ at the end loss analyzers maps magnetically to the $16 \mathrm{~cm}$ limiter radius of the central cell.

rable to the electron energy confinement time previously observed in Phaedrus plasmas with such relatively collisional electrons [17], [18].

\section{CONCLUSION}

We have implemented a whistler electron cyclotron emission diagnostic in the end cell of the Phaedrus-B tandem mirror. The emission appears to have a thermal component which is blackbody, as theory predicts, in as much as good agreement is obtained with gridded end loss energy analyzer data. The WECE shows significant electron heating due to the end-cell ICRF sources. Since the end loss data show that these temperature changes occur predominantly at the edge, it appears that the WECE diagnostic is sensitive to emission from the entire plasma cross section. Short bursts of emission many times larger than this thermal signal are also seen, suggestive of an instability.

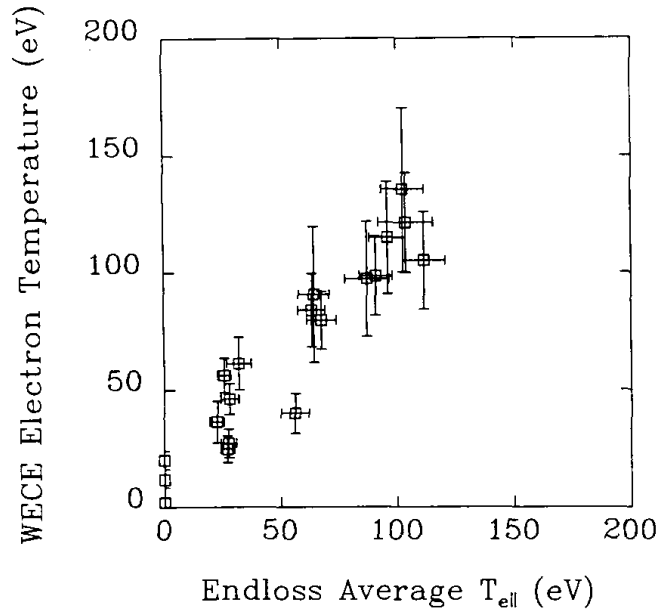

Fig. 13. WECE electron temperature versus the east end loss-energy-analyzer electron temperature. The error bars for the WECE data are the standard deviations in a 24 shot average of the signal after the bursts were removed numerically, and for the end loss data they are computed in the fitting procedure, which converts the swept data to temperatures.

\section{REFERENCES}

[1] B. A. Trubnikov and V. S. Kudryartsev, "Plasma radiation in a mag netic field," in Proc. 2nd U.N. Cong. Peaceful Uses of Atomic Energy (Geneva), 1958, vol. 31, p. 93.

[2] G. Bekefi, Radiation Processes In Plasmas. New York: Wiley, 1966.

[3] D. A. Boyd, F. J. Stauffer, and A. W. Trivelpiece, "Synchrotron radiation from the ATC tokamak plasma," Phys. Rev. Lett., vol. 37, p. $98,1976$.

[4] F. Engelmann and M. Curatolo, "Cyclotron radiation from a rarefied inhomogeneous magnetoplasma," Nucl. Fusion, vol. 13, p. 497, 1973.

[5] R. F. Ellis, G. D. Tsakiris, and D. A. Boyd, "Whistler-made electron cyclotron emission in a mirror plasma," Phys. Rev. Lett., vol. 48, p. 93, 1982.

[6] G. D. Tsakiris and R. F. Ellis, "Measurements of Budden tunneling parallel to a magnetic field,'" Phys. Rev. Lett., vol. 49, p. 874, 1982.

[7] G. D. Tsakiris and R. F. Ellis, "Whistler-mode electron cyclotron emission as an electron temperature diagnostic for magnetic mirror devices,"' Nucl. Fusion, vol. 23, p. 1115, 1983.

[8] T. A. Casper, Y. J. Chen, R. F. Ellis, R. James, and C. J. Lasnier, "Assessment of hot electron microstability in the initial TMX-U experiment," Lawrence Livermore Nat. Lab., Livermore, CA, Rep. UCID-1978, 1983.

[9] C. J. Lasnier et al., "TMX-U electron cyclotron emission measurements and modeling," Bull. Amer. Phys. Soc., vol. 28, p. 1117, 1983.

[10] R. C. Garner et al., "Hot electron microinstabilities in the Constance B mirror experiment," Bull. Amer. Phys. Soc., vol. 30, p. 1489, 1985.

[11] D. E. Baldwin and B. G. Logan, "Improved tandem mirror fusion reactor," Phys. Rev. Lett., vol. 43, p. 1318, 1979.

[12] N. Hershkowitz et al., "Enhancement of the plasma potential by fluctuating electric fields near the ion cyclotron frequency," Phys. Rev. Lett., vol. 55 , p. $947,1985$.

[13] Phaedrus Group, "Results of the phaedrus programme," Nucl. Fusion, vol. 26, p. 1213, 1985.

[14] R. A. Breun et al., "Stabilization of MHD modes in an axisymmetric magnetic mirror by applied RF waves and initial results of PhaedrusB," in Plasma Physics and Controlled Nuclear Fusion Research 1986 (Proc. 1lth Int. Conf. Kyoto, 1986). Vienna: IAEA, 1985, vol. 2, p. 265 .

[15] R. H. Goulding, "RF startup in the Phaedrus and Phaedrus-B tandem mirrors," Ph.D. dissertation, Univ. of Wisconsin, Madison, 1987.

[16] R. F. Ellis, G. D. Tsakiris, and D. A. Boyd, "'Emission, absorption, and tunneling of whistler waves in an inhomogeneous magnetic field," Phys. Fluids, vol. 26, p. 1528, 1983. 
[17] D. L. Smatlak, "Electron heat conduction in the Phaedrus tandem mirror," Ph.D. dissertation, Univ. of Wisconsin, Madison, 1982.

[18] H. Persing, private communication 1988.

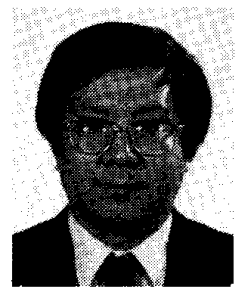

Binsheng Cui was born in Harbin, the People's Republic of China, on September 21, 1951. He graduated from the Department of Physics, Peking University, the People's Republic of China, in 1978.

He worked at the Division of Plasma Physics, Institute of Physics, at the Chinese Academy of Sciences from 1978 to 1986 . Since September 1986 he has been studying WECE diagnostics as a Research Assistant with the Phaedrus Program currently a Ph.D. candidate there. at the University of Wisconsin-Madison, and is

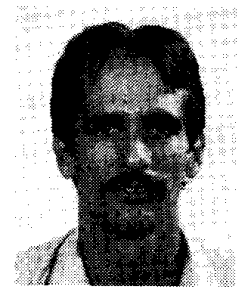

Paul H. Probert was born in Milwaukee, WI, on October 20, 1952. He received the B.S. degree in 1975 , the M.S. degree in 1976, and the Ph.D. de gree in 1985, all in electrical engineering from the University of Wisconsin-Madison. He worked at the Torsatron-Stellarator Laboratory as a Postdoctoral Researcher from 1985 to 1987.

Currently, he is an Assistant Scientist working with ECH and diagnostics in the Phaedrus Program of the Nuclear Engineering and Engineering Physics Department at the University of Wiscon-

sin-Madison

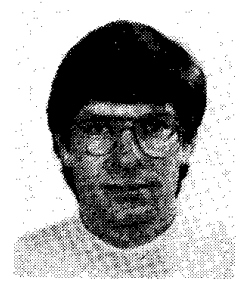

Peter B. Brooker was born in San Mateo, CA on October 23, 1958. He received the B.S. degree in physics from the University of Manitoba. Winnipeg, MB, Can., in 1981 and the M.S. degree in muclear engineering and engineering physics from the University of Wisconsin-Madison in 1984 . He expects to receive the $\mathrm{Ph}$. D. degree in nuclear engineering and engineering physics from the University of Wisconsin-Madison in 1989

His research work in the Phaedrus Program of the Department of Nuclear Engineering and Engineering Physics at the University of Wisconsin-Madison deals with the relationship of electron temperature profiles to axial potential profiles in the Phaedrus-B tandem mirror.

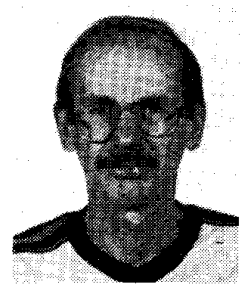

Paul D. Nonn was born in Budapest, Hungary, on March 28, 1939. He studied physics, mathamatics, and geology at the University of Wisconsin-Madison from 1960 to 1967

He worked from 1967 to 1978 at the Physics Department at the University of Wisconsin-Madison on high-power microwave and RF sources. Since 1978 , he has been working at the Nuclear Engineering and Engineering Physics Department, University of Wisconsin-Madison on RF and microwave sources and plasma diagnostics.

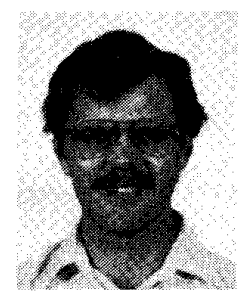

Douglas L. Keil was born in New Berlin, WI, on May 28, 1959. He received the M.S. degree in physics in 1985, from the University of Wisconsin-Madison where he is currently a Ph.D. candidate

He has been working with the Phaedrus Program at the University of Wisconsin-Madison since 1985. Within this group, he has been investigating the physics of electron heating in the electron cyclotron range of frequencies in the end cells of the Phaedrus-B tandem mirror.

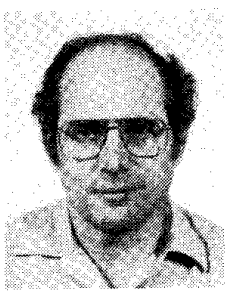

Noah Hershkowitz (SM'82) was born in Brooklyn, NY, on August 16, 1941. He received the B.S. degree (physics) from Union College, Schenectady, NY, and the Ph.D. degree (physics) from The Johns Hopkins University, Baltimore, MD, in 1966

He worked as an Instructor at The Johns Hop kins University until he joined the University of Iowa as an Assistant Professor in 1967. From 1967-1971 he carried out Mössbauer effect studies of nuclear physics and magnetism, and from 1971-1981 he worked with experimental basic plasma physics. During leaves from the University of Iowa, he spent 1974-1975 and 1980-1981 at the University of California at Los Angeles and the University of Colorado. Since 1981 he has been Professor of Nuclear Engineering and Engineering Physics and Director of the Phaedrus Program at the University of Wisconsin-Madison. His current research is a combination of ICRF fusionrelated physics and basic plasma physics. His experience includes ion acoustic solitons, electrostatic shocks, double layers, sheaths, emissive probes, electrostatic confinement in tandem mirrors, and ponderomotive force from ICRF. He has authored over 140 journal articles.

Dr. Hershkowitz served as an Associate Editor of Physics of Fluids from 1981-1983 and is currently a Divisional Associate Editor of Physical Review Letters. In 1985 he was president of the University Fusion Association. He has served on program committees for several American Physical Society (APS) meetings and is a Fellow of the APS. In 1987 he received the IEEE Nuclear and Plasma Sciences Society Merit Award. He is currently on the IEEE Nuclear and Plasma Sciences Society Administrative Committee.

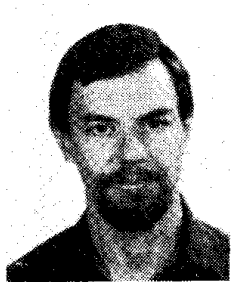

Richard P. Majeski was born on March 14, 1952 in Plainfield, NJ. He received the B.S. degree in physics in 1973, and the M.S. degree in physics in 1974, both from the University of Scranton, Scranton, PA. He earned the Ph.D. degree in plasma physics from Dartmouth College, Hanover, $\mathrm{NH}$, in 1979.

From 1979 until 1985 he held a Research Position at the University of Maryland Laboratory for Plasma Research. Since 1985, he has been an Associate Scientist in the Nuclear Engineering and Engineering Physics Department at the University of Wisconsin-Madison, investigating ICRF effects. He is primarily interested in the effects associated with ICRF heating at plasmas in both mirror devices and tokamaks.

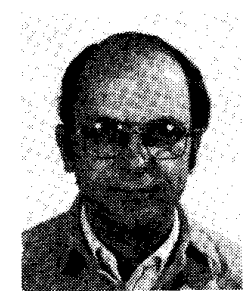

Robert A. Breun was born in Minneapolis, MN on September 25, 1945. He received the B.S. degree (physics) from the University of Minnesota, Minneapolis, in 1967, and the M.S. degree (physics) in 1968 and Ph.D. degree (physics) in 1975. both from the University of Wisconsin-Madison.

From 1975 to 1977 he worked as Adjunct Assistant Professor, Assistant Scientist, and Laboratory Manager for Prof. A. Y. Wong at the University of California, Los Angeles. Since 1977 he has been working with the Phaedrus Program of the Department of Nuclear Engineering and Engineering Physics at the University of Wisconsin-Madison and is currently Senior Scientist and Assistant Project Director. His current experimental interests include radial diffusion in an RF heated tandem mirror, edge RF effects in a tokamak, and carbon layers generated by methane-hydrogen plasmas.

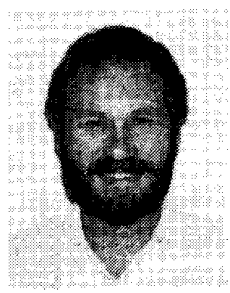

Richard F. Ellis was born on October 9, 1944, in New York City. He graduated from Cornell University, Ithaca, NY, in 1966 with the B.A. degree in physics, and eamed his Ph.D. degree in plasma physics from Princeton University, Princeton, NJ, in 1970 .

He served as a Guest Scientist at the Max Planck Institute for Plasma Physics, a Scientific Staff member at the Los Alamos National Laboratory, a Faculty Member at Dartmouth College, and a Staff Physicist at the Lawrence Livermore National Laboratory. Since 1979 he has been a Faculty Member of the Physics Department and the Laboratory for Plasma Research at the University of Maryland at College Park. 